\title{
A NEW APPROACH TO MEASUREMENT OF SACCHARIFYING CAPACITIES OF CRUDE CELLULASE
}

\author{
Bin $\mathrm{Wu}^{\mathrm{a}}$, Yue Zhao ${ }^{\mathrm{a}}$, Pei Ji Gao ${ }^{\mathrm{a}^{*}}$ \\ A practical, quantitative approach has been designed, which makes it \\ possible to accurately estimate the saccharifying activities of crude \\ cellulase preparations for insoluble cellulosics. The challenge in activity \\ determination imposed by changes in hydrolysis time and concentration \\ of cellulase and cellulosics on the assay could be overcome by selection \\ of the specific conversion percentage of cellulose as a function of \\ cellulase concentration, that is, the hydrolysis percentage of filter paper \\ by unit cellulase per minute, as the objective function with respect to \\ different concentrations of crude cellulase. A rational and governing \\ equation for crude cellulase assay was derived, and reliable results for \\ quantitatively estimating the saccharifying activities of crude cellulases \\ during the progress of hydrolysis of several cellulosic substrates were \\ obtained.
}

Keywords: Cellulase; Cellulose; Conversion; Hydrolysis

Contact information: a: The State Key Laboratory of Microbial Technology, Shandong University, Jinan, 250100, China; *Corresponding author:Gaopj@sdu.edu.cn

\section{INTRODUCTION}

Crude cellulases are composed of multi-component enzyme mixtures, which can be classified into three types: endoglucanase (1,4- $\beta$-D-glucan glucohydrolase EC 3.2.1.4), exoglucanase (1,4- $\beta$-D-glucan cellobiohydrolase, EC 3.3.1.19), and $\beta$-glucosidase ( $\beta$-D-glucoside glucohydrolase EC 3.2.1.21). A synergistic action of these enzymes is necessary for the complete hydrolysis of crystalline cellulose (Beguin and Aubert 1994). The hydrolysis rate is a compound function with reaction conditions that mainly depend on the ratio of the endo- to exo-glucanase, the concentrations of cellulase and cellulose, and the hydrolysis time. The reaction rate continuously declines as the conversion percentage of cellulose increases, and the specific rate at a given percentage of conversion also decreases with increasing cellulose concentration (Sharrock 1988). Because of the complexity of the cellulase-cellulose system, it is difficult to quantitatively describe the reaction rate and mechanism; thus, the kinetic characteristics in the initial reaction period and in the extended stages are significantly different, compared with the homogeneous enzyme catalytic reaction system, so that a MichaelisMenten type rate expression cannot be applied to those enzymatic hydrolysis reactions. As Sharrock suggested, "the measurement of cellulase activity must be approached using somewhat uncertain techniques" (Sharrock 1988).

A variety of assays and substrates have been used for measurements of cellulase activities. These can be grouped into two basic approaches with respect to activity: One is based on the determination of the individual activity of the cellulase component; 
whereas the other is based on the evaluation of the total saccharifying capacity of a crude cellulase system for the hydrolysis of certain cellulosics (Sharrock 1988). Until now, accurately determining cellulase activities, however, has been limited to cases with a single cellulase component with a soluble substrate (Wood and Bhat 1998; Day and Workman 1984; Deshpande et al. 1984; Manning 1981; Hagerman et al.1985; Wu et al. 2006).

A series of assay procedures has been used for estimating crude cellulase activities on insoluble cellulose. The activities of cellulase are commonly evaluated by endpoint analysis of the products, using a reducing sugars assay, and therefore, results are expressed as the saccharifying capacity. However, most of this work is not readily expressed in a quantitative manner, lacks a theoretical basis, and does not take into account all the effective factors, such as the concentration of cellulose and cellulase, the hydrolysis time, the ratio of crystalline and amorphous cellulose, and the proportion between different components in the enzyme preparation. These methods are neither rapid nor readily adaptable to kinetic measurements (Sharrock 1988). Thus, the applicability of those methods is sometimes limited to certain hydrolysis conditions; in fact, there is a considerable confusion inherent in the enzyme names, units and activities (Sharrock 1988; Deshpande et al. 1984; Manning 1981). Mandels and Webber proposed a filter paper assay (FPA) method for identifying the saccharifying activity of a cellulase preparation from cellulolytic fungi (Mandels and Webber 1969). Although this method has been recommended by the International Union of Pure and Applied Chemists (IUPAC) for evaluation of potential saccharifying capacity of a cellulase system (Ghose 1987), it has been long recognized for its complexity and susceptibility to operator error, and has a reputation for not being reproducible. Since then, some improved FPA (filter paper assay) methods have been proposed to improve the reproducibility of the filter paper assay, most of which are limited to improvements in sugar estimation (Griffin 1973; Kelly et al. 2003; Gao 1987); thus, the problem of an accurate and reproducible saccharifying assay for cellulase still remains.

In this study, a new approach to accurately estimating the potential capacity of a cellulase system for the hydrolysis of insoluble cellulosics is described.

\section{EXPERIMENTAL}

\section{Materials and Methods}

\section{Cellulolytic substrates}

Filter paper (Whatman No.1), which was adopted for the present work, is composed of crystalline and amorphous cellulose. De-waxed cotton fibers, composed of 95\% cellulose, were used to represent highly crystalline native cellulose (DP about 1000). Crystalline cellulose (Avicel) PH101 was obtained from SERVA (DP about 200). Phosphate swelling cellulose, prepared by $85 \%$ phosphate acid swelling method (Wood and Bhat 1998), represented amorphous cellulose. 


\section{Production and purification of cellulase}

A cellulolytic fungus, Trichoderma pseudokoningii S-38, was isolated previously by our laboratory and used for production of cellulase (Ma 1990). Enzyme production, purification and determination of its activity were performed as described in previous reports (Yan and Sun 1997; Yan et al. 1997).

Hydrolysis of cellulose samples by crude cellulase

The hydrolysis assay procedure was performed in $25 \mathrm{~mL}$ flasks that contained different concentrations of cellulose substrate (w/v) suspended in $5 \mathrm{~mL}$ (final volume) $\mathrm{pH}$ 4.8, $50 \mathrm{mM}$ acetate buffer, to which was added the crude cellulase solutions. Hydrolysis was performed at $45^{\circ} \mathrm{C}$ in a shaking bath at $15 \mathrm{rpm}$ (adding $0.001 \% \mathrm{NaN} 3 \mathrm{w} / \mathrm{v}$ to prevent contamination). Every two hours, the mixture was centrifuged at 5,000 g for $10 \mathrm{~min}$ to separate the hydrolyzate. These samples were analyzed for reducing sugars content in the supernatant by the dinitrosalicylic acid (DNS) method (Ghose 1987).

\section{Effects of cellulase and cellulose concentration on the hydrolysis kinetics}

In general, the rate at which cellulase hydrolyzes cellulose can be defined in two ways. One is based on the number of reducing end groups, usually expressed as an equivalent weight of glucose, produced per unit volume of cellulose suspension after application of a defined amount of cellulase after a prescribed incubation time. This will be called the apparent hydrolysis rate. The second way is as the equivalent weight of glucose reducing end groups produced in the cellulose suspension per unit of time and per unit of cellulose (weight or volume) at a selected enzyme level.

\section{RESULTS AND DISCUSSION}

\section{Results of the Hydrolysis Process}

Filter paper was chosen as a typical substrate for cellulase catalysis assays, because it has both crystalline and amorphous fractions, moderate susceptibility to hydrolysis, and availability as a convenient and reproducible substrate.

Fig. 1 shows a typical time course for a fixed amount of filter paper $(0.2 \mathrm{mg} / \mathrm{mL})$ hydrolyzed by different concentrations of cellulase $(0.25 \mathrm{~mL}$ to $1.25 \mathrm{~mL}$ added $)$, while Fig. 2 shows a similar time course for different concentrations of filter paper ( 0.08 to 0.4 $\mathrm{mg} / \mathrm{mL})$ hydrolyzed by a fixed amount of cellulase $(0.25 \mathrm{~mL})$.

The results in Figs. 1 and 2 appear to be in good agreement with many published sets of data in this field (Beguin and Aubert 1994; Eriksson et al. 2002; Zhang et al. 1999; Valjamae et al. 1998; Ortega et al. 2001). All of the results led to the conclusion that as the more digestible material becomes consumed, the cellulose remaining was more and more difficult to be hydrolyzed. The hydrolysis rate depended on the concentration of cellulase and cellulose. Both the condition of the cellulose and the concentration of cellulase change with time during the course of an experiment. This was indicated, since the objective variable (reducing sugars formed) was a compound function, which increased with the hydrolysis time and the concentration of cellulase and cellulose. 


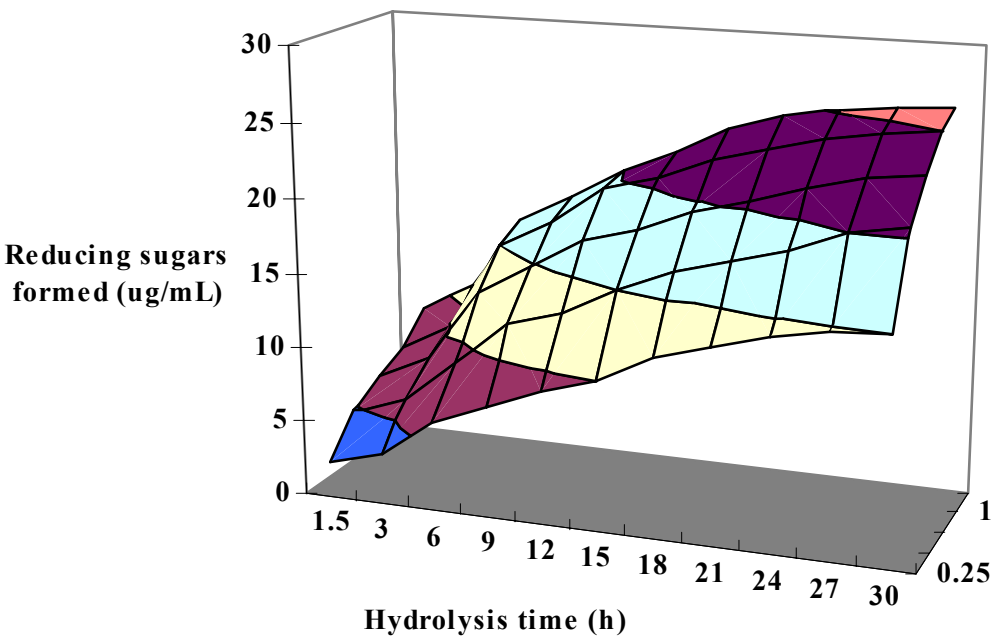

Cellulase added

(mL)

Fig. 1. Time course of hydrolysis curves for filter paper $(0.2 \mathrm{mg} / \mathrm{mL})$ by different concentrations of crude cellulase from Trichoderma pseudokoningii S-38

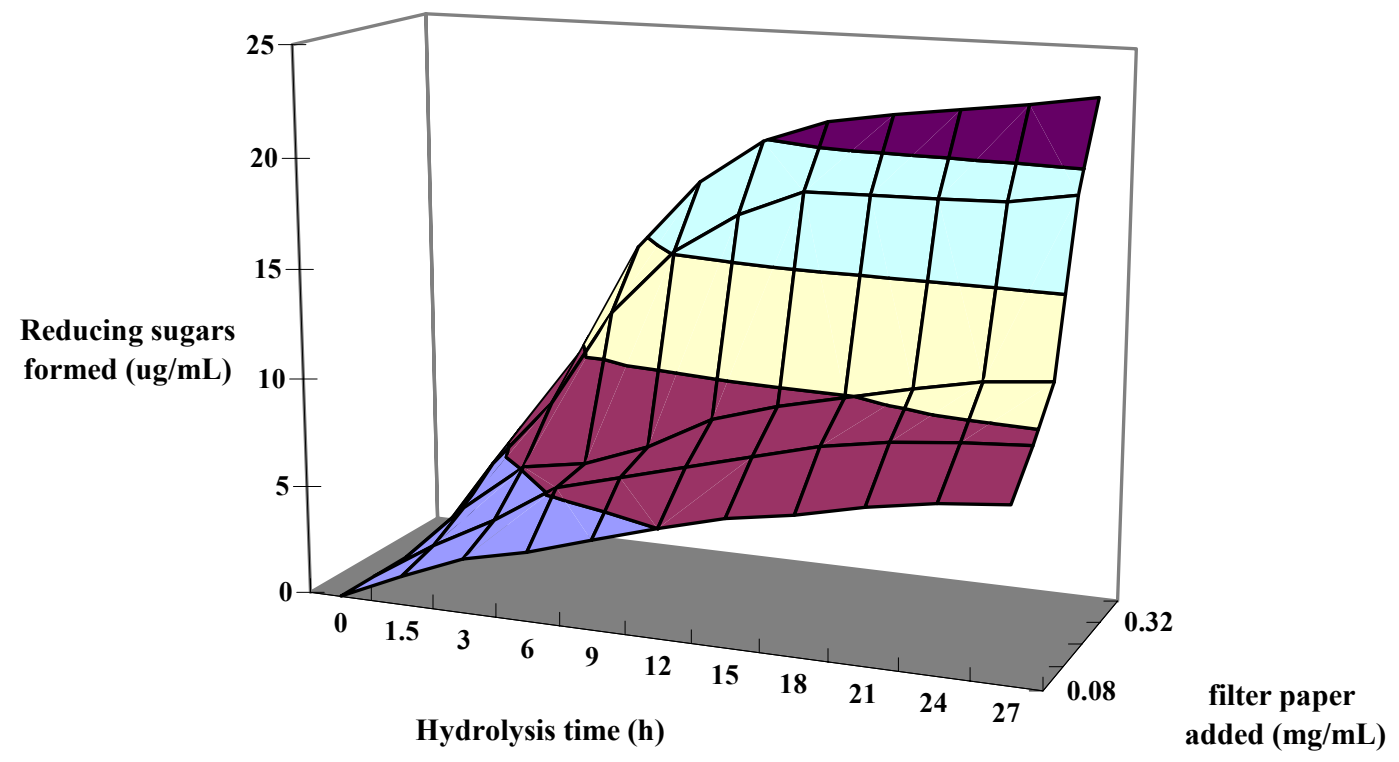

Fig. 2. Time courses of reducing sugars produced during hydrolysis of different concentration of filter paper by crude cellulase $(0.25 \mathrm{~mL})$ 
Under the conditions used, linear relationships between activities of enzyme and the cellulase added only appeared within a narrow range of the curves. Only the data in such ranges could be fitted to the linear equations. Significant effects on specific activity were observed only when considering relatively low concentrations of cellulase and relatively short hydrolysis times (Figs. 3 and 4). The specific activity was calculated as follows:

$$
\text { Specific activity }=\frac{\text { Reducing sugar formed }}{\mathrm{t} \times[\text { cellulase }][\text { cellulose }]}
$$

where, $\mathrm{t}$ is the hydrolysis time, [cellulase] is the concentration of cellulase added, and [cellulose] is the concentration of cellulose added. Under these conditions, the majority of hydrolyzed cellulose is the amorphous fraction; thus, the intrinsic saccharifying capacity for cellulase cannot be accurately estimated based on those shorter hydrolysis times.

In Figs. 3 and 4, the plotted points represent the observed data, and the lines are the fitting results obtained by application of the one-phase exponential decay equation. The parameter $\mathrm{R}^{2}$ is the coefficient of determination in the goodness of fit test.

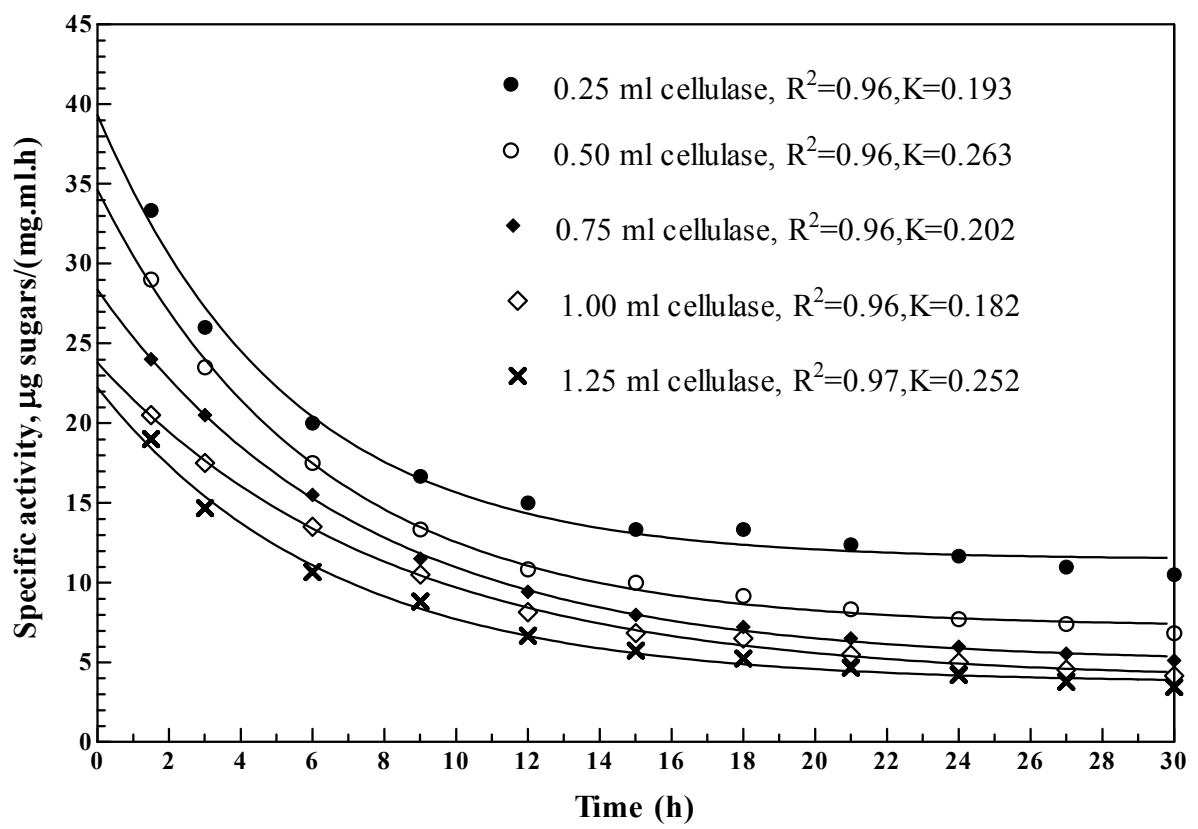

Fig. 3. Changes of the specific activities of cellulase during hydrolysis of $0.2 \mathrm{mg} / \mathrm{mL}$ cellulose at different concentrations of crude cellulase (0.25-1.25 mL) (data derived from Fig. 1). 


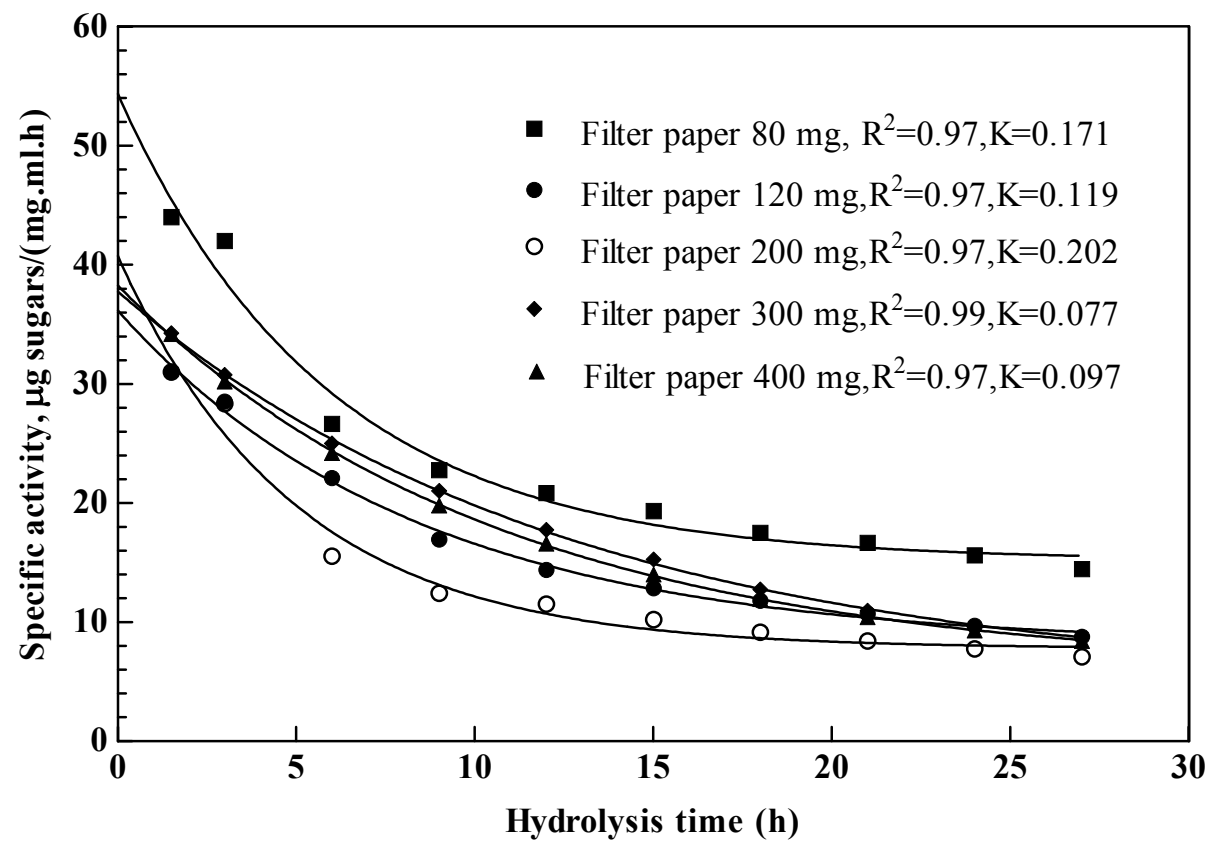

Fig. 4. Changes of the specific activities of cellulase during hydrolysis at different concentrations of filter paper $(0.08-0.4 \mathrm{mg} / \mathrm{mL}$ ) by $0.25 \mathrm{~mL}$ of crude cellulase (data derived from Fig. 2).

\section{Relationship of Hydrolysis Rate and Conversion Percent of Cellulosics during Hydrolysis Process}

As mentioned above, in order to accurately estimate the potential capacity of a crude cellulase sample in the hydrolysis of insoluble cellulosics, the substrates must be hydrolyzed to a certain extent to show the synergism of all the cellulase components. As reported in our previous study, quantitatively estimating the activity behavior of cellulase during cellulose hydrolysis can be elucidated by multiple regression analysis (Wang et al. 2004). But this method is generally regarded as being applicable only for pure cellulase components, and is inadequate for crude cellulases.

For the present estimation, a new parameter, substrate conversion percentage (SC), was introduced. It was defined as the ratio between the cellulosics hydrolyzed $\left(\mathrm{S}_{0}-\mathrm{S}\right.$, where " $\mathrm{S}$ " is the quantity of cellulosics remaining) and the original cellulosics $\left(\mathrm{S}_{0}\right)$ :

$$
\mathrm{SC}=\frac{\mathrm{S}_{0}-\mathrm{S}}{\mathrm{S}_{0}} \times 100 \%
$$

When results of the present study are viewed in combination with other accumulated reports (Mandels and Webber 1969; Ghose 1987; Lynd 2002), the combined data suggest that SC always increases during the progress of hydrolysis (Fig. 5), and the experimental data also produced a hyperbolic shape curve. 


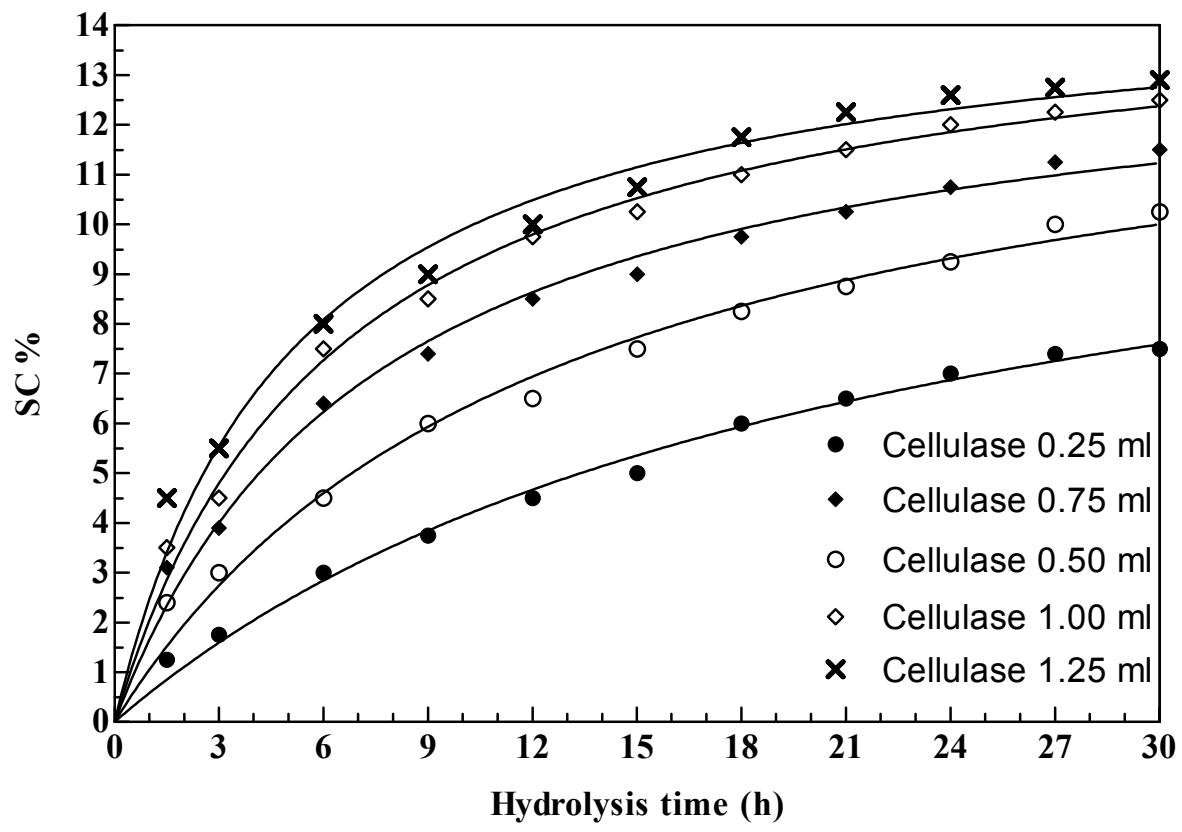

Fig. 5. Time courses of SC in the hydrolysis by different concentrations of cellulase (Data derived from Fig. 1)

The reaction rates all decreased continuously with hydrolysis time and increasing cellulase concentration. Based on these results, estimation of the cellulase activity is complex, because the accurate estimation of reactive value at each point required the combination of calculating the partial derivative of $\mathrm{SC}(\mathrm{Y})$ with respect to time $(\mathrm{t})$ and cellulose concentration $(\mathrm{C})$, respectively, as

$$
\mathrm{dY}=\frac{\partial \mathrm{Y}}{\partial \mathrm{C}} \mathrm{dC}+\frac{\partial \mathrm{Y}}{\partial \mathrm{t}} \mathrm{dt}
$$

However, the calculation of the partial derivatives of $\mathrm{Y}$ with respect to $\mathrm{C}$ and $\mathrm{t}$ is very intricate.

We considered that two factors simultaneously caused the changes of SC during the progress of hydrolysis: first, the heterogeneous structure of cellulose, second, the cellulase reacting may be quantitatively responsible for the changes of Specific Substrate Conversion percentage (SSC). This parameter is calculated similarly to specific activity, and the only difference is that the SC replaces the value of reducing sugar formed.

$$
\mathrm{SSC}=\frac{\mathrm{SC}}{\mathrm{t} \times[\text { cellulase }][\text { cellulose }]}
$$

where $t$ is the hydrolysis time, [cellulase] is the concentration of cellulase added, and [cellulose] is the concentration of cellulose added. Time courses of SSC are shown in Fig.6. 


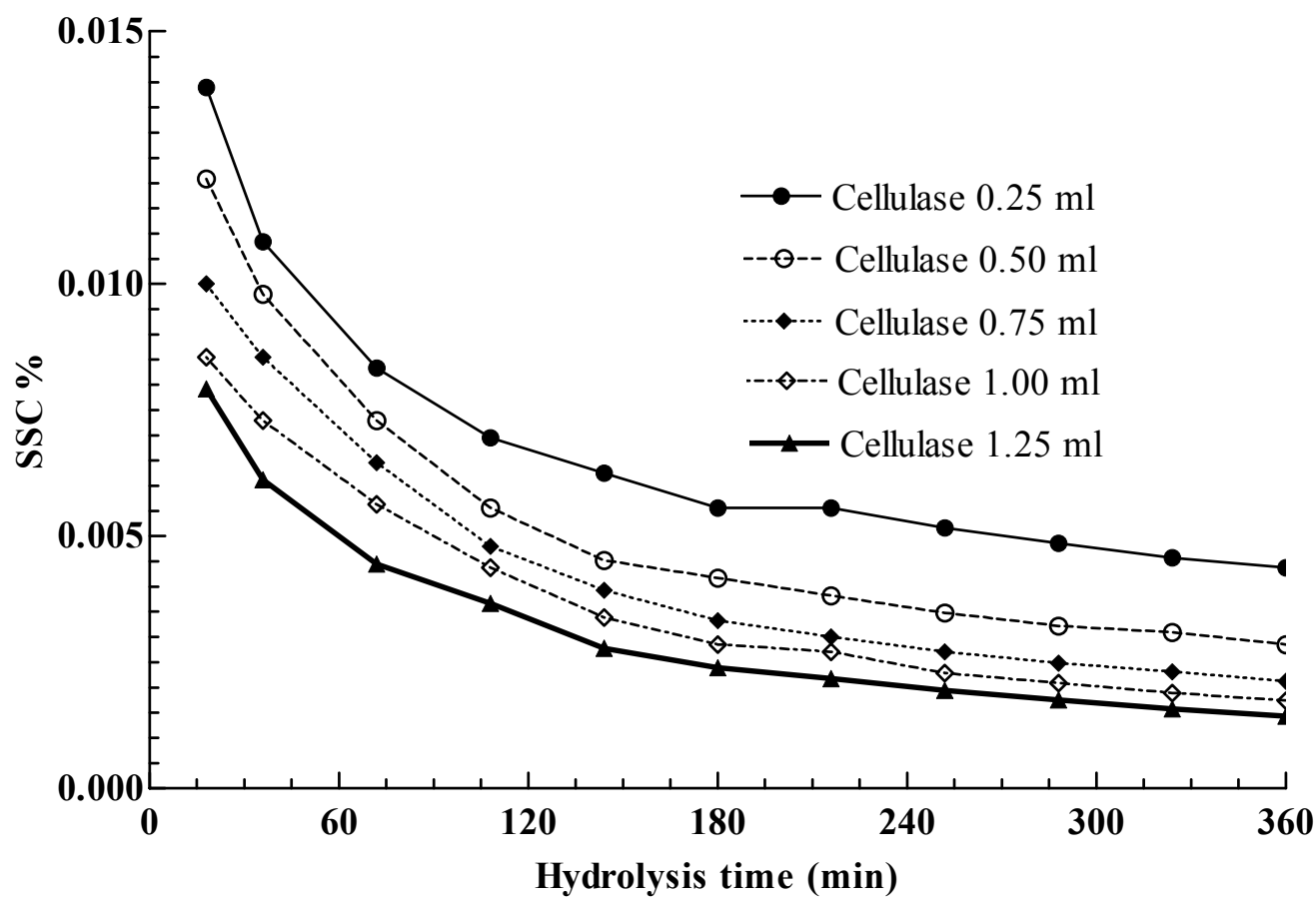

Fig. 6. Time courses of SSC (data derived from Fig. 5)

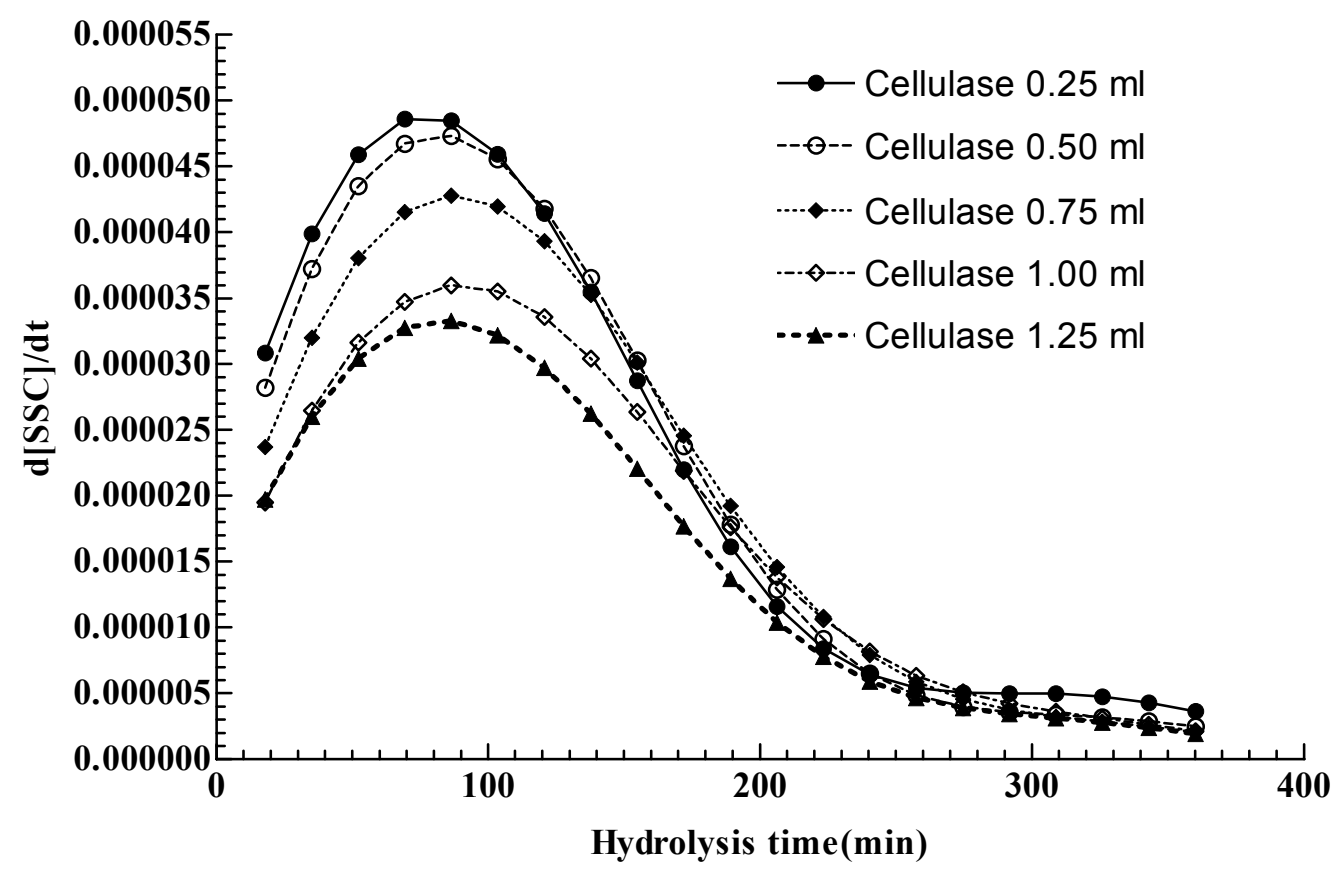

Fig. 7. Instantaneous rate curves of $\mathrm{d}[\mathrm{SSC}] / \mathrm{dt}$ for different cellulase concentrations and hydrolysis times 
The critical index for evaluation in enzymatic capacity, according to the traditional approach, is its initial velocity; this can be solved by the instantaneous rates (Wu 2006). Figure 7 shows the instantaneous rates plot of $d[S S C] / d t$ versus time under different cellulase concentrations. It appears as a bell-shaped curve, but with different kinetic characteristics and cannot be directly fit by a linear or non-linear equation that applies to the entire progress of reaction.

As mentioned above, for an insoluble and heterogeneous substrate - the cellulose - to expresses intrinsic catalytic capacity, the entire hydrolysis information is necessary. So we used the AUC method (the total area under the curve) for evaluation of cellulase capacity (Fig. 8). This is a common nonlinear method used in pharmacokinetics (Perrier 1982).

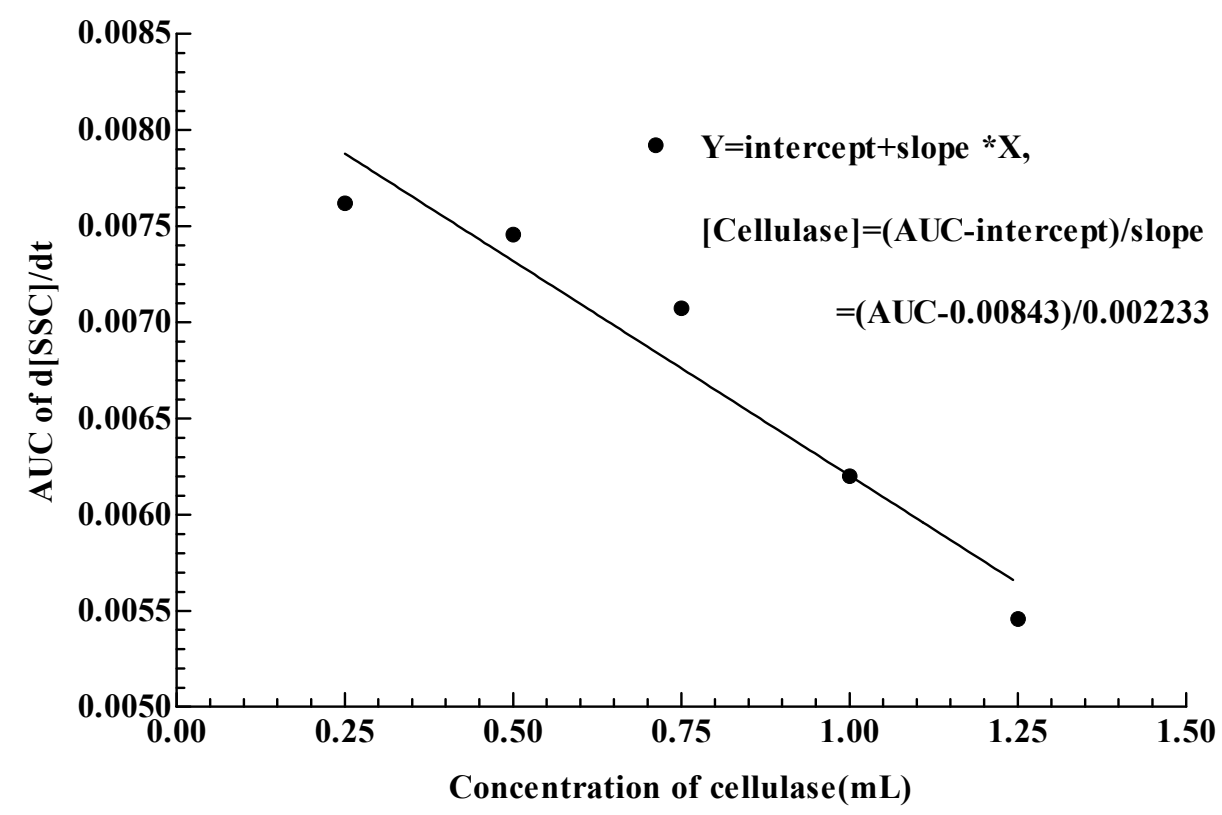

Fig. 8. The relationship of cellulase concentration and its AUC calculated as $d[S S C] / d t$

Reliable results were obtained, as the Specific·SC (SSC) varied in a wide range. The cellulose hydrolysis ranged from 1.5 to $12.5 \%$ (W/V) for different concentrations of cellulase, according to which a rational and simple equation for crude cellulase assay was obtained:

$$
\mathrm{Y}=\text { intercept }+ \text { slope } * \mathrm{X}
$$

in which $\mathrm{Y}$ is the AUC of $\mathrm{d}[\mathrm{SSC}] / \mathrm{dt}$, and $\mathrm{X}$ is the concentration of cellulase.

As might be expected, the intrinsic saccharifying capacity for a crude cellulase can be obtained by this approach, because the effects of three factors - concentration of cellulase, cellulose, and hydrolysis time - are all considered in this equation. The slope of the linear equation could be represented as the total saccharifying capacity of a crude 
cellulase sample under experimental conditions corresponding to the design of a practical hydrolysis process for insoluble cellulosics.

Similar results were obtained when choosing other cellulosics such as cotton fibers, microcrystalline cellulose, and phosphate swelling cellulose as substrates (data not shown).

\section{Summary of Assay Procedure}

The overall assay procedure, in summary, consists of the following steps:

\section{Hydrolysis of cellulose samples by crude cellulase}

Different concentrations of cellulose substrate are hydrolyzed by different concentrations of crude cellulase solutions. In general, five combinations for cellulose and cellulase with different concentrations would be enough, and the reducing sugars are determined every two hours. The total hydrolysis time is varied based on the structure of cellulosics; for example, for filter paper 12 hours is enough and for cotton fibers it may be 24 hours.

\section{Calculation of the evaluation of cellulase capacity}

Calculate the value of SC (substrate conversion percentage) and SSC (specific substrate conversion percentage), and convert the data to obtain AUC (the total area under the curve) for evaluation of cellulase capacity. Data analysis can be easily performed by Microsoft Excel and the graphical software Prism 6.0 or other public software.

\section{ACKNOWLEDGMENTS}

The authors are grateful for the support of a Grant from National Basic Research Program of China (No. 2003 CB716006 and No. 2004 CB 719702), and the Natural Science Foundation of Shandong (No.Y2004D09).

\section{REFERENCES CITED}

Beguin, P., and Aubert, J. P. (1994). "The biological degradation of cellulose," FEMS Microbiology Reviews 13, 25-58.

Day, D. F., and Workman, W. E. (1982). "A kinetic assay for cellulases," Anal. Biochem. 126, 205-207.

Deshpande, M. V., Eriksson, K. E., and Pettersson, L. G. (1984) "An assay for selective determination of exo-1,4,- $\beta$-glucanases in a mixture of cellulolytic enzymes," Anal. Biochem. 138, 481-487.

Eriksson, T., Karlsson, J., and Tjerneld, F. (2002). "A model explaining declining rate in hydrolysis of lignocellulose substrates with cellobiohydrolase (cel 17A) and endoglucanase I (cel 17B) of Trichoderma reesei," Appl. Biochem. Biotechnol. 101, 41-60. 
Gao, P. J. (1987). "A simple method for estimating cellobiose activity by determination of reduced sugars," Biotech. \& Bioeng. 24, 903-905.

Ghose, T K. (1987). Measurement of cellulase activities. Pure \& Appl. Chem. 59, $257-$ 268.

Griffin, H. L. (1973). "Filter paper assay: Effect of time and substrate concentration on cellulase activity," Anal. Biochem. 56, 621-625.

Hagerman, A. E., Blau, D. M. and McClure, A. L. (1985). "Plate assay for determining the time of production of protease, cellulase, and pectinases by germinating fungal spores," Anal. Biochem. 151, 334-342.

Kelly, G. C., Mazzari, C. A., Kim, S., et al. (2003). "Suggested improvements to the standard filter paper assay used to measure cellulase activity," Biotechnol. and Bioeng. 82, 745-749.

Lynd, L. R., Wrimer, P. J., Zyl, W. H. (2002). "Pretornus is microbial cellulose utilization: fundamental and biotechnology," Microbiology and Molecular Biology Reviews 66, 506-577.

Ma, D. B., Gao, P. J., and Wang, Z. N. (1990). "Preliminary studies on the mechanism of cellulose formation by Trichoderma pseudokoningii S-38," Enzyme Microb. Technol. 12, 631-635.

Mandels, R., and Webber, J. (1969). The production of cellulose, In: Advan. Chem. Washington DC, 391-413.

Manning, K. (1981). "Improved viscometric assay for cellulase," J. Biochem. Biophys. Methods 5(4), 189-202.

Ortega, N., Busto, M. D., and Mateob, M. P. (2001). "Kinetics of cellulose saccharification by Trichoderma reesei cellulases," International Biodetermination and Biodegradation 47, 7-14.

Perrier, M. G. D. (1982). Pharmacokinetics, 2nd ed. Marcel Dekker, New York.

Sharrock, K. R. (1988). "Cellulase assay method: a review," J. Biochem. and Biophys. Methods 17, 81-106.

Valjamae, P., Sild, V., Pettersson, G. et al. (1998). "The initial kinetics of hydrolysis of hydrolysis by cellobiohydrolase I and II is consistent with a cellulose surface-erosion model," Eur. J. Biochem. 253, 469-475.

Wang, L. S., Zhang, Y. Z. and Gao, P. J. (2004). "Quantitative estimate of the effect of cellulase components during degradation of cotton fibers," Carbohydrate research 339(4), 819-824.

Wood, T. M., and Bhat, K. M. (1998). Methods for cellulase activities In W. A. Wood, and S. T. Kellong, Ed., Methods in Enzymology Academic Press, Inc San Diego, California, 92101. vol. 160 part A.

Wu, B., Zhao, Y., and Gao, P. J. (2006). "Estimation of cellobiohydrolysis I activity by numerical differentiation of dynamic ultraviolet spectroscopy," Acta Biochim. Biophys. Sin. 38, 372-378.

Yan, B. X., \& Sun, Y. Q. (1997). "Domain structure and conformation of a cellobiohydrolase I from Trichoderma pseudokoningii S-38," J. Protein. Chem. 16,59-66.

Yan, B. X., Sun, Y. Q., and Gao, P. J. (1997). "Intrinsic fluorescence in endoglucanase and cellobiohydrolase from Trichoderma reesei disrupting the structure of cellulose," J. Protein Chem.18, 681-688. 
Zhang, S., Dolfgang, D. E., and Wilson, D. B. (1999). "Substrate heterogeneity causes the nonlinear kinetics of insoluble cellulose hydrolysis," Biotech. \& Bioeng. 66,35-41.

Article submitted: May 31, 2006; Revisions accepted: September 13, 2006; Published: September 21, 2006; Correction to Figs. 5, 6, 7, and 8 uploaded Oct. 3, 2006. 